\title{
Optimizing PAT data transmission: assessing the accuracy of temperature summary data to estimate environmental conditions
}

\author{
Bauer Robert ${ }^{1, *}$, Forget Fabien ${ }^{1,2}$, Fromentin Jean-Marc ${ }^{1}$ \\ ${ }^{1}$ Univ Montpellier, CNRS, Ifremer,UMR Marbec, IRD,CS 30171, F-34203 Sete, France. \\ 2 SAIAB, Grahamstown, South Africa. \\ * Corresponding author : Robert Bauer, email address : rbauer@gmx.com
}

\begin{abstract}
:
Pop-up archival tags (PAT) provide summary and high-resolution time series data at predefined temporal intervals. The limited battery capabilities of PATs often restrict the transmission success and thus temporal coverage of both data products. While summary data are usually less affected by this problem, as a result of its lower size, it might be less informative. We here investigate the accuracy and feasibility of using temperature at depth summary data provided by PATs to describe encountered oceanographic conditions. Interpolated temperature at depth summary data was found to provide accurate estimates of three major thermal water column structure indicators: thermocline depth, stratification and ocean heat content. Such indicators are useful for the interpretation of the tagged animal's horizontal and vertical behaviour. The accuracy of these indicators was found to be particularly sensitive to the number of data points available in the first $100 \mathrm{~m}$, which in turn depends on the vertical behaviour of the tagged animal. Based on our results, we recommend the use of temperature at depth summary data as opposed to temperature time series data for PAT studies; doing so during the tag programming will help to maximize the amount of transmitted time series data for other key data types such as light levels and depth.
\end{abstract}

Keywords : mixed layer depth, ocean heat content, pop-up archival tags, PAT-style Depth-Temperature Profiles, temperature at depth, thermal stratification, thermocline

\section{Introduction}

Pop-up archival tags (PAT) have enabled scientists to study the movement and habitat use of large marine predators including tunas, billfishes and sharks. Archival tags do not only provide position estimates (via geolocation) but also ins-situ depth and temperature measurements. This information gives valuable insight into the animals' movement and encountered physical oceanographic conditions (Biuw et al., 2007; Costa et al., 2008).

PATs generally provide two types of data i) high resolution time series-and ii) summary data at predefined temporal intervals. The data transmission via the ARGOS (Advanced Research and Global Observation Satellite) system creates a significant bottleneck owing to limited satellite coverage and 
the PATs battery life. Due to its fine temporal resolution, times series data represents far more data than summary data. Hence, transmitted summary data is usually more complete, achieving a better temporal coverage of the study period. Several factors affect the amount of transmitted data and thus the achieved temporal coverage: 1) the number of data types to be transmitted (i.e. depth, temperature, light, etc), 2) their sampling temporal resolution, and 3) the tag deployment duration.

It is therefore important to clearly define the research questions, in order to optimize the later data transmission and coverage already during the tag programming. In this context, the transmission of temperature time series data is often switched off and sacrificed for the study of large scale movements and vertical behavior. However, temperature summary data is always reported by PATs. This data has been previously used to infer sea surface temperature, thermocline and mixed layer depth (Teo et al., 2007; Howell et al., 2010; Abecassis et al., 2012). Although temperature time series data can provide accurate estimates of the thermal water column structure (Costa et al., 2008), the accuracy of such estimates is yet to bet tested for temperature summary data.

Oceanographic conditions are of high interest for studies that investigate potential structuring elements of the vertical behaviour of marine animals. This applies in particular to the thermal water column structure, notably the thermocline which represents the transition zone between the warmer surface waters (mixed later) and the colder deep water layer. This zone is characterized by a strong temperature and density gradient (Fiedler, 2010), that serve as an aggregation zone for particulate matter, giving it a significant biological importance across different trophic levels (Pelletier et al., 2012; Walli et al., 2009).

Other important indicators of the thermal water column structure include the thermal stratification and ocean heat content. The thermal stratification represents a measure of the water column stability that can complement indicators the thermocline. The ocean heat content is the energy contained in the water column up to the depth with a specified temperature threshold (Leipper and Volgenau, 1972). Thermal water column structure indicators can help differentiate thermal properties between water masses even when sea surface temperature gradients are low (e.g. in the tropics). This makes them an interesting input for PAT geolocation models that use reference oceanographic fields to refine position estimates (e.g. Shay and Brewster (2010) give an approach to estimate the ocean heat content from satellite data).

In this study, we investigate the accuracy and applicability of using PAT temperature at depth summary data to describe encountered oceanographic conditions. More precisely, we tested the performance of such summary data to estimate the three major indicators of the thermal water column structure- the thermocline depth, the thermal stratification and the ocean heat content.

\section{Material and Methods}

\section{Temperature summary data}

High-resolution time series data can be summarized in several ways. For example, PATs from "Wildlife Computers Inc.” (MK10 and MiniPAT tags; www.wildlifecomputers.com, Redmond, Washington, USA), calculate temperature at depth summary data throughout the deployment period, known as "PAT-style Depth-Temperature Profiles" (PDT).

This data provides the minimum and maximum temperatures at different depth intervals, during a userdefined summary period (1--24h), which is also used for other summary data products (e.g. time at depth and time at temperature frequencies). To do so, the tag bins the recorded depth time series data to 
a $8 \mathrm{~m}$ resolution (Wildlife Computers, personal communication). However, to improve transmission efficiency, only a subset of this binned data is transmitted with the corresponding minimum and maximum temperatures, by default 8 depth bins. This number can be increased to 16, when a high resolution option is selected during the tag setup, but this mode operates only when the tag reaches depths greater than $400 \mathrm{~m}$ during the summary period (fixed manufacturer setting). It must be noted that due to recent changes in the firmware the high resolution mode is now no longer optional in the tag setup but always selected. We performed our analysis with both low and high resolution modes, as the former is still relevant to many conducted tagging studies. To select the bins to be transmitted, the tag splits the binned data into 8-16 equally spaced "regions", of which the most popular depth bins are transmitted. The minimum and maximum depth bins are thereby always reported.

We used the complete time series data (temporal resolution of $10 \mathrm{~s}$ ), obtained from a recovered MK10 PAT, deployed on an Atlantic bluefin tuna in the Northwestern Mediterranean Sea (162 days; Fromentin and Lopuszanski, 2013), to calculate low and high resolution daily PDT data. In order to test the accuracy and performance of the PDT datasets, we used the same time series data to calculate daily average temperatures for all possible $8 \mathrm{~m}$ bins. This dataset was then used as a reference for comparative analyses with both PDT datasets. Since average temperatures are not reported by the PDT data, we conducted a preliminary analysis, on the correlation and differences, between daily average temperatures and the average of daily minimum and maximum temperatures (henceforth called "average PDT-temperature”) for the entire $8 \mathrm{~m}$ binned dataset.

\section{Vertical temperature interpolation}

Daily average temperatures of the low and high resolution PDT datasets as well as daily average temperatures of the binned time series dataset were linearly interpolated to produce continuous daily temperature profiles with a $0.5 \mathrm{~m}$ grid resolution. We used the binned time series data with a $8 \mathrm{~m}$ resolution instead of the raw time series data $(0.5 \mathrm{~m}$ resolution) to increase the number of records per depth for the calculation of average temperatures, thereby removing excessive noise from the daily temperature profiles. We then evaluated the accuracy of the interpolated profiles of both PDT datasets by testing their correlation against profiles of the interpolated $8 \mathrm{~m}$ binned reference time series data. To be more stringent, we only tested data points within the first $100 \mathrm{~m}$ where most of the thermal structure was observed.

\section{Thermal water columns structure comparisons}

\section{Thermocline and mixed layer depth}

The thermocline depth was estimated using the "Maximum slope by difference"-method (Fiedler, 2010). This method defines the thermocline as the depth segment with the maximum negative temperature gradient (-dT/dz) between all temperature pairs spaced by dz $\geq 20 \mathrm{~m}$. For each dataset ( $8 \mathrm{~m}$ binned time series data, low and high resolution PDT), we calculated the temperature gradients of all $20 \mathrm{~m}$ segments per daily interpolated temperature profile. Thermocline depths were then estimated 
using the average depth of the segment(s) corresponding to the maximum negative slope. Note that the mixed layer depth can be considered as the lowest depth of these segments, marking the beginning of the thermocline zone (Fiedler, 2010). Due to the correlation between thermocline and mixed layer depth, the latter was not considered in this study.

\section{Thermal stratification and ocean heat content}

As an indicator of the thermal stratification, we used the standard deviation of daily interpolated temperature profiles per dataset up to a depth of 150 m (Valdés and Moral, 1998).

The ocean heat content (OHC) is defined by

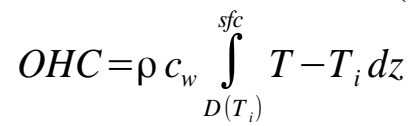

quantifying the energy of a temperature profile $T$ between the ocean surface (sfc) and the deepest depth (D) recorded for a fixed temperature threshold $\left(T_{i}\right)$, with $\rho$ representing the density of water and $c_{w}$ the specific heat capacity of sea water (Meyers et al., 2014).

We applied a temperature threshold of $15^{\circ} \mathrm{C}$ and a fixed specific heat capacity of $3994.6 \mathrm{~J} \mathrm{~kg}^{-1} \mathrm{C}^{-1}$ for seawater for OHC estimations. Water density per depth was calculated using the "swRho"-function of the R-package “oce” (Kelley and Richards, 2015), assuming a fixed salinity of 35 PSU.

\section{Results}

\section{Average temperature vs average PDT-temperature}

Average temperatures and average PDT-temperatures of the $8 \mathrm{~m}$ binned time series data were highly correlated $\left(R^{2}=0.989, p \leq 0.01\right.$; Fig. 1$)$. Differences between both datasets were minor and ranged mostly between -0.5 to $0.5^{\circ} \mathrm{C}(98.5 \%$ of data; Fig. 1$)$.

\section{Vertical interpolation}

Daily interpolated profiles of all three datasets show large similarities despite their different resolutions (Fig. 2). This was further confirmed by the high correlation between interpolated profiles of both PDT datasets and those of the reference time series data (Tab. 1). The high resolution PDT obtained a slightly higher correlation $\left(R^{2}=0.97, p \leq 0.01\right.$; Tab. 1) than the low resolution PDT. Differences between the two PDT datasets were particularly evident during September when the low resolution PDT data appears to be less accurate in estimating the thermal water column structure owing to the large depth range exhibited by the tagged tuna during that time. 


\section{Thermal water column structure indicators}

All three thermal water column structure indicators estimated from the low and high resolution PDT data exhibited similar trends to those of the reference time series dataset (Fig. 3). Amongst the indicators tested, the thermocline showed a slightly lower correlation and thus appears to be more sensitive to the vertical resolution of the PDT data (Tab. 1). The average difference between thermocline estimates of the low and high resolution PDT datasets and those of the reference time series was $-6.3 \mathrm{~m}$ (SD+/- $31.2 \mathrm{~m}$; 76.68\% of absolute differences $\leq 20 \mathrm{~m})$ and $-5.3 \mathrm{~m}(\mathrm{SD}+/-29.3 \mathrm{~m}$; $82.8 \%$ of absolute differences $\leq 20 \mathrm{~m}$ ), respectively.

Largest differences between the estimates were found during winter months when the thermal gradients are particularly weak (e.g. January, see Fig. 1) and the thermocline is non-existent. Thermal stratification and ocean heat content indices were more sensitive to the PDT resolution during days of strong thermal stratification (e.g. September, see Fig. 3).
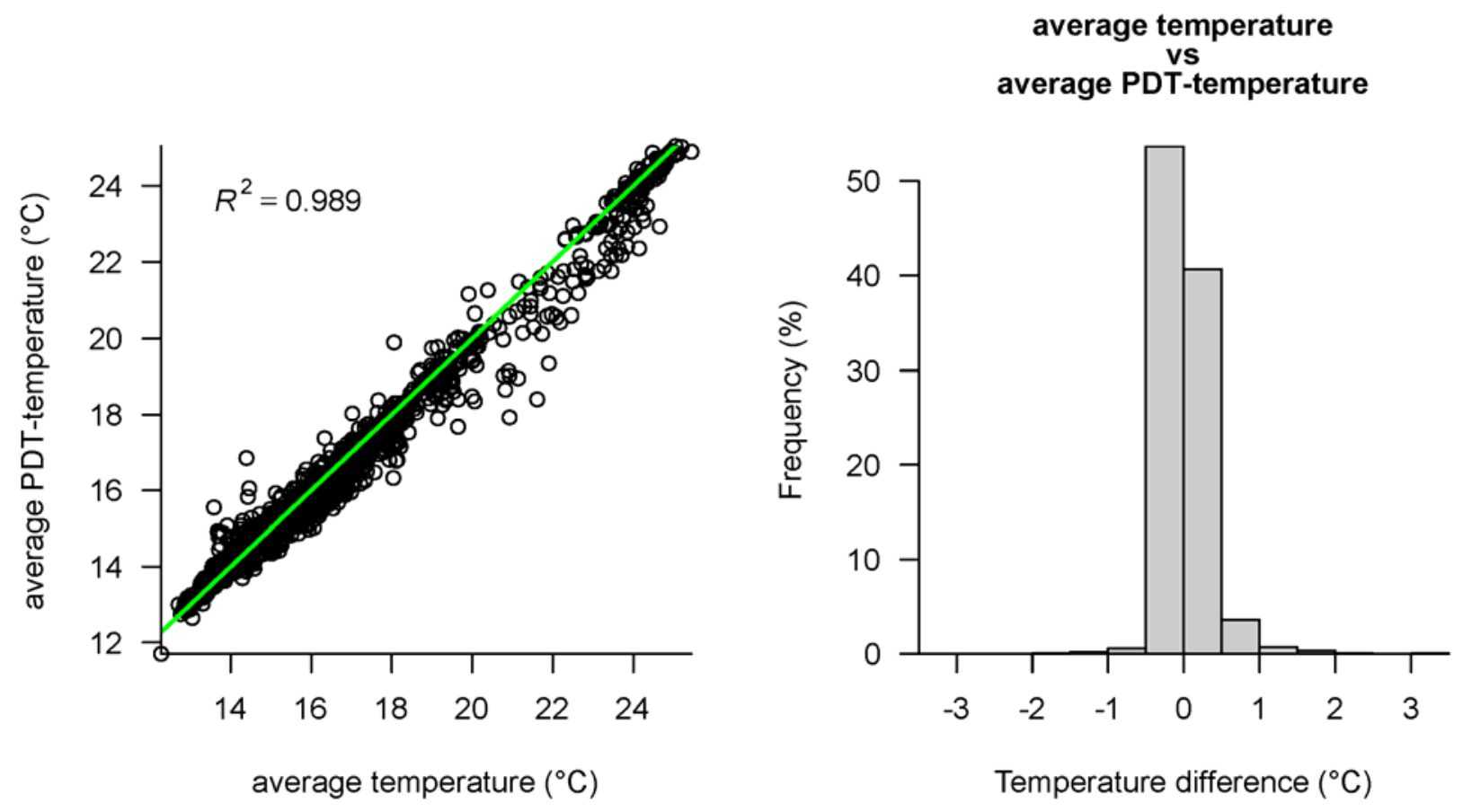

Fig.1: Correlation (left) and differences (right) between average temperatures and average PDTtemperatures of all possible depth intervals of the $8 \mathrm{~m}$ binned time series dataset. The green line indicates a 1:1 correlation. In total 4611 data points are compared. 

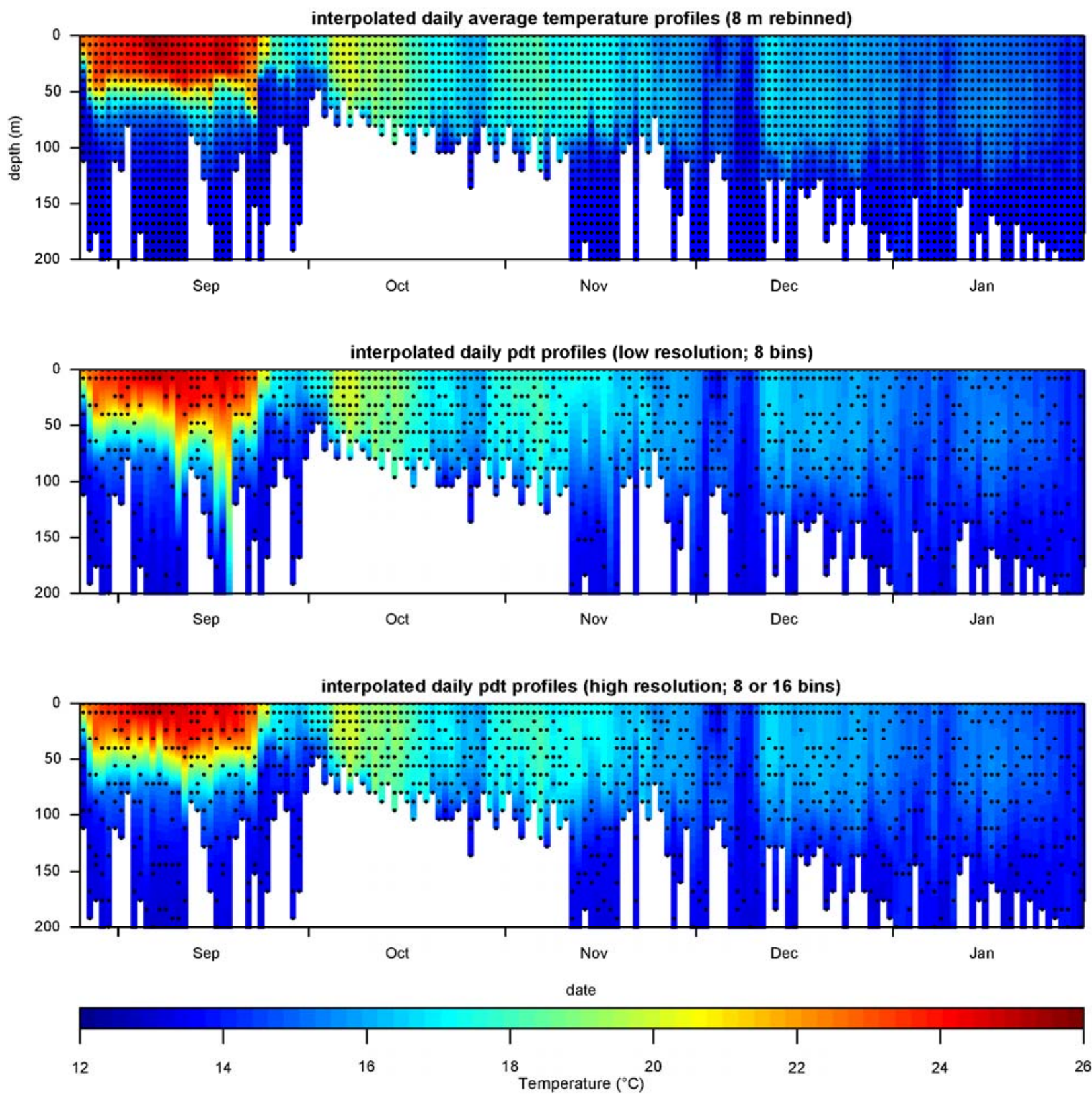

Fig.2: Daily interpolated temperature profiles for binned time series data (average temperature per 8 $\mathrm{m}$ ), low (8 bins) and high resolution (8 or 16 bins) PDT data for the first $200 \mathrm{~m}$. The black dots indicate data points for each dataset. 

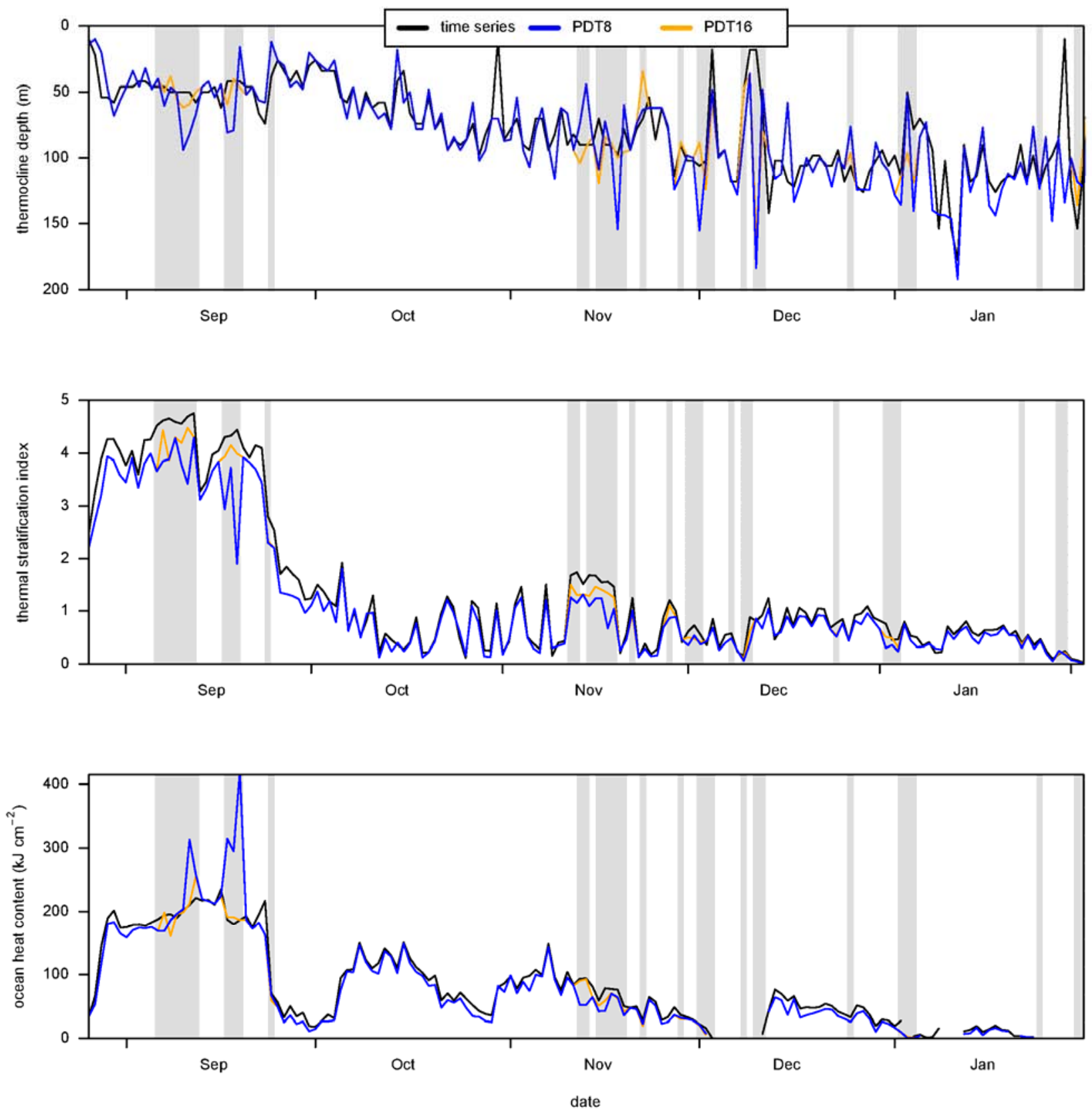

Fig.3: Time series of thermal water column structure indicators (from top to bottom: thermocline depth, thermal stratification index and ocean heat content) estimated from interpolated profiles of binned time series data (black lines) and PDT data (orange line: low resolution, blue line: high resolution). Grey bars indicate dates when the tag went below $400 \mathrm{~m}$ and thus estimates of the low and high resolution PDT data potentially differ. Corresponding correlation coefficients of the comparisons are given in Tab. 1. 


\begin{tabular}{lll}
\hline & \multicolumn{2}{l}{ Correlation Coefficient } \\
\cline { 2 - 3 } & Low resolution PDT & High resolution PDT \\
Interpolation & $0.92^{*}$ & $0.97^{*}$ \\
Thermocline & $0.68^{*}$ & $0.71^{*}$ \\
Stratification Index & $0.98^{*}$ & $0.99^{*}$ \\
Ocean heat content & $0.89^{*}$ & $0.96^{*}$ \\
\hline
\end{tabular}

$* p \leq 0.01$

Tab.1: Pearson correlation coefficients of interpolated data points and thermal water column structure indicators (thermocline depth, thermal stratification index, $\mathrm{OHC} \geq 15^{\circ} \mathrm{C}$ ) of the binned time series data and both PDT data sets.

\section{Discussion}

In this study we assessed the accuracy and suitability of temperature at depth summary data (PDT) to estimate thermal water column structure indicators. The interpolation of summary data provided very similar results to that of time series data. Only 8 to 16 temperature data points were sufficient to characterize the thermal structure of the water column over several hundred meters. While it is generally desirable to collect high resolution data, our results indicate that summary data can provide a robust estimation of the thermal water column structure. Estimates tend to be more reliable when at least 4 data points are available within the first $100 \mathrm{~m}$ (depth at which most of the thermal structure is usually observed).

Due to its smaller size, the success rate of transmitted PDT data is usually higher than that of time series data, especially in areas with known transmission problems through the ARGOS system (e.g. Mediterranean sea; Fromentin, 2010). Given the limited battery capacities of PATs, we suggest using PDT data as an alternative to temperature time series data to describe the thermal water column structure. Doing so during the tag programming will reduce the total amount of tag data to be transmitted and thus maximize the amount of transmitted time series data for other key data products such as light levels and vertical data. More precisely, this would save the transmission of 144-1152 temperature time series data points per day, depending on the selected time series resolution (1.25-10 $\min )$.

An improvement in the accuracy of interpolated PDT temperature profiles and derived water column structure indicators could be achieved by increasing the temporal resolution of the summary data during the tag setup (e.g. from 24 to $12 \mathrm{~h}$ ). This will essentially increase the sampling and might be particularly useful if the studied species is known to exhibit distinct diel vertical behaviour patterns. 


\section{Acknowledgements}

The authors would like to thank Matt Rutishauser from Wildlife Computers for helping to better understand the PDT calculation process. RKB was supported by a $\mathrm{PhD}$ grant from France Filière Pêche ( $\mathrm{N}^{\circ}$ LM-2012-144) and IFREMER (fellowship contract to RKB). Tagging was funded by the Ifremer research program "DEMOSTEM”.

\section{References:}

Abecassis, M., Dewar, H., Hawn, D., Polovina, J. (2012) Modeling swordfish daytime vertical habitat in the North Pacific Ocean from pop-up archival tags. Mar. Ecol. Prog. Ser. 452:219-236.

Biuw, M., Boehme, L., Guinet, C., Hindell, M., Costa, D., Charrassin, J.B., Roquet, F., Bailleul, F., Meredith, M., Thorpe, S., Tremblay, Y., McDonald, B., Park, Y.H., Rintoul, S.R., Bindoff, N., Goebel, M., Crocker, D., Lovell, P., Nicholson, J., Monks, F., Fedak, M.A. (2007) Variations in behavior and condition of a Southern Ocean top predator in relation to in situ oceanographic conditions. Proc. Natl. Acad. Sci. USA. 104(34):13705-13710.

Costa, D.P., Klinck, J.M., Hofmann, E.E., Dinniman, M.S., and Burns, J.M (2008) Upper ocean variability in west Antarctic Peninsula continental shelf waters as measured using instrumented seals. Deep-Sea Res. Pt. II Top. Stud. Oceanogr. 55(3-4):323-337.

Fiedler, P.C. (2010) Comparison of objective descriptions of the thermocline. Limnol. Oceanogr-Meth. 8:313-325.

Fromentin, J.M. (2010) Tagging bluefin tuna in the Mediterranean Sea: challenge or mission: impossible? Collect. Vol. Sci. Pap. ICCAT. 65(3):812-821.

Fromentin, J.M. and Lopuszanski, D. (2013) Migration, residency, and homing of bluefin tuna in the western Mediterranean Sea. ICES J. Mar. Sci. 71(3):510-518.

Howell, E.A., Hawn, D.R., and Polovina, J.J. (2010) Spatiotemporal variability in bigeye tuna (Thunnus obesus) dive behavior in the central North Pacific Ocean. Prog. Oceanogr. 86(1-2):8193.

Kelley, D., \& Richards, C. (2015) oce: Analysis of Oceanographic Data. http://cran.rproject.org/package $=$ oce

Leipper, D.F. and Volgenau, D. (1972) Hurricane Hear Potential of the Gulf of Mexico. J. Phys. Oceanogr. 2:218-224

Meyers, P. C., Shay, L. K., and Brewster, J. K. (2014) Development and Analysis of the Systematically Merged Atlantic Regional Temperature and Salinity Climatology for Oceanic Heat Content Estimates. J. Atmos. Ocean. Tech., 31(1):131-149.

Pelletier, L., Kato, A., Chiaradia., A., and Ropert-Coudert, Y. (2012) Can thermoclines be a cue to prey distribution for marine top predators? A case study with little penguins. PLOS ONE 7(4):4-8. 
Shay, L.K. and Brewster, J.K. (2010) Oceanic Heat Content Variability in the Eastern Pacific Ocean for Hurricane Intensity Forecasting. Mon. Weather Rev. 138(6):2110-2131.

Teo, S.L.H., Boustany, A., Dewar, H., Stokesbury, M.J.W., Weng, K.C., Beemer, S., Seitz, A.C., Farwell, C.J., Prince, E.D., and Block, B.A. (2007) Annual migrations, diving behavior, and thermal biology of Atlantic bluefin tuna, Thunnus thynnus, on their Gulf of Mexico breeding grounds. Mar. Biol. 151:1-18.

Valdés, L. and Moral, M. (1998) Time-series analysis of copepod diversity and species richness in the southern Bay of Biscay off Santander, Spain, in relation to environmental conditions. ICES J. Mar. $\begin{array}{lll}\text { Sci. } & \text { 55(4)., 783-792. }\end{array}$

Walli, A., Teo, S.L.H., Boustany, A., Farwell, C.J., Williams, T., Dewar, H., Prince, E., and Block, B.A. (2009) Seasonal movements., aggregations and diving behavior of Atlantic bluefin tuna (Thunnus thynnus) revealed with archival tags. PloS One. doi:10.1371/journal.pone.0006151 